\title{
Correction to: Low cost and sustainable hyaluronic acid production in a manufacturing platform based on Bacillus subtilis 3NA strain
}

\author{
Sebastián Cerminati ${ }^{1}\left[\right.$ ( Mélanie Leroux ${ }^{2} \cdot$ Pablo Anselmi $^{1} \cdot$ Salvador Peirú ${ }^{1} \cdot$ Juan C. Alonso $^{3} \cdot$ Bernard Priem $^{2}$. \\ Hugo G. Menzella ${ }^{1}$
}

Published online: 23 August 2021

๑) Springer-Verlag GmbH Germany, part of Springer Nature 2021

Correction to: Applied Microbiology and Biotechnology (2021) 105:3075-3086 https://doi.org/10.1007/s00253-021-11246-6

The published version contains a mistake.

In Table 2, the cost " $\$ 12.86$ " in row "Manufacturing cost per kg”, column 2, should read “\$ 112.86”.

Publisher's note Springer Nature remains neutral with regard to jurisdictional claims in published maps and institutional affiliations.

The original article can be found online at https://doi.org/10.1007/ s00253-021-11246-6

Sebastián Cerminati

cerminati@iprobyq-conicet.gob.ar;

sebastiancerminati@gmail.com

1 Instituto de Procesos Biotecnológicos y Químicos

(IPROBYQ), Facultad de Ciencias Bioquímicas y

Farmacéuticas, Universidad Nacional de Rosario (UNR),

CONICET, Suipacha 570, S2002LRL Rosario, Argentina

2 Centre de Recherches sur les Macromolécules Végétales, CNRS, Université Grenoble Alpes, 38041 Grenoble, France

3 Department of Microbial Biotechnology, Centro Nacional de Biotecnología, CNB-CSIC, 28049 Madrid, Spain 